\title{
In vitro activity of piperacillin/tazobactam and ertapenem against Bacteroides fragilis and Escherichia coli in pure and mixed cultures
}

\author{
Kênia Valéria dos Santos, Cláudio Galuppo Diniz, \\ Simone Cristina Coutinho, Ana Carolina Morais Apolônio, \\ Luciana Geralda de Sousa-Gaia, Jacques Robert Nicoli, \\ Luiz de Macêdo Farias and Maria Auxiliadora Roque de Carvalho
}

\begin{abstract}
Correspondence
Maria Auxiliadora Roque de

Carvalho

marc@icb.ufmg.br
\end{abstract}

Received 7 December 2006

Accepted 18 February 2007
Departamento de Microbiologia, Instituto de Ciências Biológicas, Universidade Federal de Minas Gerais, Belo Horizonte, MG, Brazil

\begin{abstract}
Ertapenem and piperacillin/tazobactam are $\beta$-lactam antibiotics with a broad spectrum of activity used for the treatment of mixed infections in which Bacteroides fragilis and Escherichia coli play an important aetiological role. In this study, the activities of piperacillin/tazobactam and ertapenem (MIC and time-kill kinetics) against these bacteria were compared. MICs were determined by the agar dilution method, and the time and slope of time-kill curves were analysed. In the in vitro pharmacodynamic assays, pure and mixed cultures of $E$. coli and $B$. fragilis were exposed to peak concentrations of ertapenem $\left(8.0 \mu \mathrm{g} \mathrm{ml}^{-1}\right)$ and piperacillin/tazobactam (64.0/8.0 $\mu \mathrm{g} \mathrm{ml}^{-1}$ ) for $48 \mathrm{~h}$. Treatment with ertapenem reduced the viability of $E$. coli and/or $B$. fragilis by 3 logs in all experiments, whereas piperacillin/tazobactam only affected the viability of $B$. fragilis. Both drugs exhibited their fastest rates of killing when bacteria were grown in mixed cultures. According to the results, ertapenem exhibited activity similar to that of piperacillin/tazobactam against $B$. fragilis alone or in mixed culture. However, ertapenem exhibited a markedly higher activity against $E$. coli alone or in combination with $B$. fragilis relative to piperacillin/tazobactam.
\end{abstract}

\section{INTRODUCTION}

Complicated intra-abdominal infections require a combination of surgery/drainage and antimicrobial therapy. The choice of antimicrobial therapy must take into account the complex normal aerobic and anaerobic microbiota of the bowel, which reach population levels of up to $10^{11}$ c.f.u. (g faeces) $^{-1}$ (Solomkin \& Konstantin, 2003). Bacteroides fragilis, an obligate anaerobe, and Escherichia coli, a facultative anaerobe, are the most frequently isolated bacteria from intra-abdominal abscesses (Goldstein \& Snydman, 2004). Thus the presence of mixed infections of anaerobic and facultative bacteria with different susceptibilities often requires the use of either dual therapy, e.g. a penicillin plus an aminoglycoside, or broad-spectrum antibiotics, such as carbapenens or $\beta$-lactam/ $\beta$-lactamase inhibitor combinations including piperacillin/tazobactam (Perry \& Markham, 1999; Solomkin \& Konstantin, 2003).

Ertapenem is a new carbapenem with a broad spectrum of activity against Gram-negative pathogens, including extended-spectrum $\beta$-lactamase- and AmpC-producing members of the Enterobacteriaceae, as well as against

Abbreviation: LLA, lower limit of accuracy.
Gram-positive aerobic and anaerobic pathogens (Goldstein \& Snydman, 2004; Wexler, 2004). Clinical trials in complicated intra-abdominal infection have demonstrated that ertapenem has equivalent efficacy and safety to piperacillin/tazobactam (Solomkin et al., 2003; Dela Pena et al., 2006).

The in vitro activities of piperacillin/tazobactam and ertapenem have been reported in MIC and time-kill studies using single cultures (Muñoz Bellido et al., 1997; Spangler et al., 1997). However, the killing of bacteria in pure culture may not be identical to the killing in mixed culture (Stearne et al., 2001; Hermsen et al., 2005; Schaumann et al., 2005).

In the present study, an anaerobic time-kill technique was used for a comparative study of the in vitro activities of piperacillin/tazobactam and ertapenem against $B$. fragilis and E. coli in pure or mixed cultures.

\section{METHODS}

Bacterial strains. B. fragilis ATCC 25285 and E. coli ATCC 25922 were used throughout the experiments. The obligate anaerobe $B$. fragilis was incubated anaerobically at $37^{\circ} \mathrm{C}$ in an anaerobic chamber 
(Forma Scientific Company) containing an atmosphere of $85 \% \mathrm{~N}_{2}$, $10 \% \mathrm{H}_{2}$ and $5 \% \mathrm{CO}_{2}$, and the facultative anaerobe E. coli was incubated aerobically or anaerobically at $37^{\circ} \mathrm{C}$.

Antibiotics. A piperacillin/tazobactam preparation (Tazocin) was purchased commercially (Lederle Piperacillin) and ertapenem sodium was kindly provided as a standard powder by Merck Sharp \& Dohme. Antibiotic solutions were prepared according to the instructions of the manufacturer.

Susceptibility testing. The MICs of the test drugs were determined by the agar dilution method using a standard inoculum $\left(\sim 10^{5}\right.$ c.f.u. $)$ and a higher inoculum size ( $\sim 10^{8}$ c.f.u.), according to protocols M7A6 and M11-A6 of the Clinical Laboratory Standards Institute (formerly the NCCLS) (NCCLS, 2003, 2004).

Time-kill curve. Time-kill curves were obtained using peak concentrations of the indicated antibiotics in serum $\left(8.0 \mu \mathrm{g} \mathrm{ml} l^{-1}\right.$ and $64.0 / 8.0 \mu \mathrm{g} \mathrm{ml}^{-1}$ for ertapenem and piperacillin/tazobactam, respectively). Prior to the time-kill curve experiments, several colonies of each bacterial strain were incubated anaerobically overnight in $10 \mathrm{ml}$ Brucella broth. Each overnight culture was then adjusted to an $\mathrm{OD}_{550}$ of 0.05 with fresh Brucella broth to yield a starting inoculum of approximately $10^{6}$ c.f.u. $\mathrm{ml}^{-1}$. This concentration was confirmed by 10 -fold serial dilution. Cultures were incubated at $37{ }^{\circ} \mathrm{C}$ in an anaerobic chamber. When the bacterial population reached the exponential growth phase (approx. $150 \mathrm{~min}$ for E. coli and $7 \mathrm{~h}$ for B. fragilis), ertapenem or piperacillin/ tazobactam solutions were added to each test vial to produce antibiotic concentrations of $8.0 \mu \mathrm{g} \mathrm{ml}^{-1}$ (ertapenem) or $64.0 / 8.0 \mu \mathrm{g}$ $\mathrm{ml}^{-1}$ (piperacillin/tazobactam). No antibiotic was added to the control vial. Cultures were then incubated at $37{ }^{\circ} \mathrm{C}$ under anaerobic conditions. These procedures were followed for both organisms during the mixed-culture experiments. In this case, when the bacterial population reached the exponential growth phase, an equal volume of each strain was added to a new vial inoculated with antibiotics, as described above. At pre-determined time points of $0,1,2,3,4,5,6,24$ and $48 \mathrm{~h}$ following the introduction of antibiotics into the vials, $1 \mathrm{ml}$ samples of the cultures were withdrawn aseptically for bacterial quantification. Bacterial counts were determined by $1: 10$ serial dilution in sterile saline and a $0.1 \mathrm{ml}$ sample of each dilution was plated onto trypticase soy agar for E. coli or Brucella agar for $B$. fragilis. In the mixed-culture experiments, MacConkey agar was used to count the number of colonies of $E$. coli after aerobic incubation for $24 \mathrm{~h}$ at $37^{\circ} \mathrm{C}$, whilst Bacteroides bile aesculin agar was used to count B. fragilis colonies after anaerobic incubation for $48 \mathrm{~h}$ at $37{ }^{\circ} \mathrm{C}$. Antibiotic carry-over was addressed by using saline dilution techniques. The lower limit of accuracy (LLA) of bacterial counting was 300 c.f.u. $\mathrm{ml}^{-1}$.

Statistical analysis. Colony count data (in $\log _{10}$ c.f.u. $\mathrm{ml}^{-1}$ ) from duplicate time-kill studies were averaged and plotted as a function of time. The slopes of the time-kill curves were compared for a rate-ofkilling analysis using a linear model with ORIGIN software (version 6.1; OriginLab Corporation). Comparison of the mean reduction in bacterial counts of the pure cultures of $B$. fragilis and E. coli with that of the corresponding mixed cultures was analysed using a two sample paired $t$-test. A $P$ value of $<0.05$ was considered to be significant.

\section{RESULTS AND DISCUSSION}

The MICs of piperacillin/tazobactam have been reported to be strongly affected by an increase in inoculum size (König et al., 1998; Betriu et al., 2006), whilst ertapenem susceptibility with high inoculum did not change (Betriu et al.,
2006). We found a moderate effect of the inoculum on the killing of both $B$. fragilis and $E$. coli as indicated by the increase in MICs when the inoculum was increased from $10^{5}$ to $10^{8}$ c.f.u. $\mathrm{ml}^{-1}$. However, these values remained below the susceptible piperacillin/tazobactam breakpoint for both bacteria (Table 1).

Time-kill curve results are presented in Figs 1 and 2 . Bacterial interactions in mixed infections can interfere with antimicrobial therapy (Heilmann, 1993; Pendland et al., 2002). In addition to $\beta$-lactamase production by bacteria involved in mixed infections, other factors such as bacteriocin production (Jerman et al., 2005) can alter bacterial susceptibility. In the present study, the bactericidal effect of ertapenem and piperacillin/tazobactam in the mixed culture was more evident than in the pure culture $(P<0.05)$. The bactericidal effect against $B$. fragilis was detected at $<2 \mathrm{~h}$ in mixed culture, but only after $6 \mathrm{~h}$ in pure culture. In addition, the number of viable $B$. fragilis cells almost reached the LLA within $6 \mathrm{~h}$ of exposure to ertapenem in mixed culture (Fig. 1). Similar data were recently described by Hermsen et al. (2005) in assays testing moxifloxacin against the same two bacteria. The time-kill curves showed that the decrease in the count of viable cells occurred more rapidly in the first $2 \mathrm{~h}$, in both pure and mixed cultures (Figs 1 and 2). Therefore, in order to determine the rate of bacterial death in this time interval, we calculated the slope of the time-kill curve by using data for the 0,1 and $2 \mathrm{~h}$ time points. All coefficients of determination in the linear regression analysis were greater than 0.93, with the exception of that for piperacillin/tazobactam against $E$. coli in mixed culture with $B$. fragilis $\left(r^{2}=0.82\right)$. This analysis confirmed that a more rapid rate of killing occurred in the mixed culture (Table 2).

The time-kill curve patterns for ertapenem and piperacillin/ tazobactam against $B$. fragilis in pure or mixed culture were similar (Fig. 1). In both cultures, ertapenem and piperacillin/tazobactam exhibited a marked bactericidal effect

Table 1. MICs $\left(\mu \mathrm{g} \mathrm{ml}^{-1}\right)$ of ertapenem and piperacillin/ tazobactam against E. coli ATCC 25922 and B. fragilis ATCC 25285

\begin{tabular}{|c|c|c|c|c|}
\hline \multirow[t]{3}{*}{ Antibiotic } & \multicolumn{4}{|c|}{ Bacterial inoculum (c.f.u. $\mathrm{ml}^{-1}$ ) } \\
\hline & \multicolumn{2}{|c|}{ E. coli } & \multicolumn{2}{|c|}{ B. fragilis } \\
\hline & $10^{5}$ & $10^{8}$ & $10^{5}$ & $10^{8}$ \\
\hline Ertapenem $^{\star}$ & 0.016 & 0.016 & 0.12 & 0.12 \\
\hline $\begin{array}{l}\text { Piperacillin/ } \\
\text { tazobactam } \dagger\end{array}$ & $2.0 / 0.25$ & $8.0 / 1$ & $0.25 / 0.03$ & $1 / 0.12$ \\
\hline
\end{tabular}

${ }^{\star}$ Breakpoints for ertapenem: B. fragilis $\leqslant 4.0 \mu \mathrm{g} \mathrm{ml}^{-1} ;$ E. coli $\leqslant 2.0 \mu \mathrm{g}$ $\mathrm{ml}^{-1}$.

$\dagger$ Breakpoints for piperacillin/tazobactam: B. fragilis $\leqslant 32.0 / 4.0 \mu \mathrm{g}$ $\mathrm{ml}^{-1}$; E. coli $\leqslant 16.0 / 4.0 \mu \mathrm{g} \mathrm{ml}^{-1}$. 

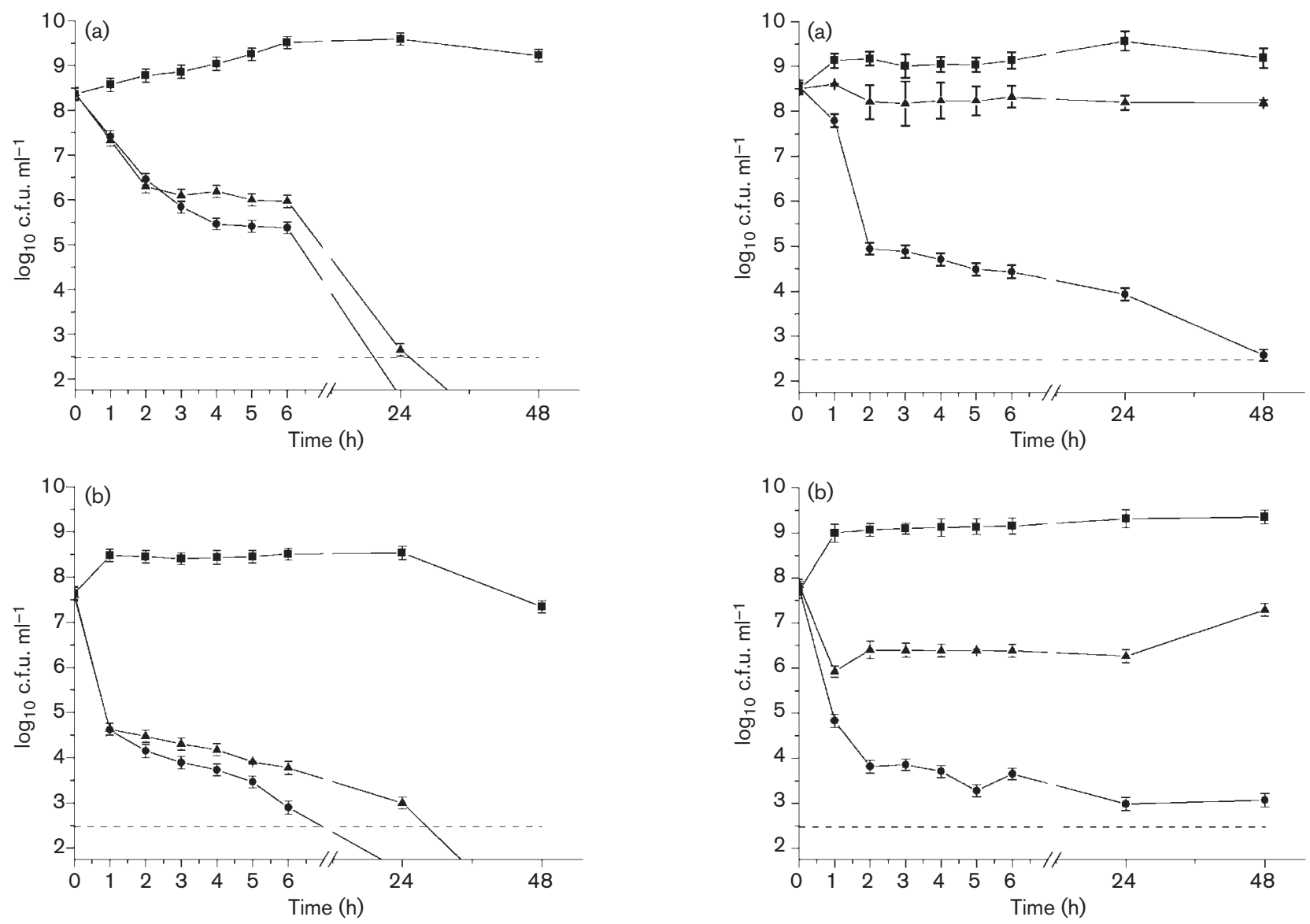

Fig. 1. Time-kill curves of ertapenem $\left(0,8.0 \mu \mathrm{g} \mathrm{ml}^{-1}\right)$ and piperacillin/tazobactam $\left(\boldsymbol{\Lambda}, 64.0 / 8.0 \mu \mathrm{g} \mathrm{m}^{-1}\right)$ against $B$. fragilis alone (a) and in mixed culture with E. coli (b). A growth control ( $\boldsymbol{\square})$ and the LLA (dashed line) are shown.

against $B$. fragilis, defined by $a \geqslant 3 \log$ decrease in the bacterial count relative to the starting inoculum, supporting their clinical use against these bacteria (Sader et al., 1998; Goldstein et al., 2000).

Ertapenem also exhibited a bactericidal effect against E. coli in both mixed and pure culture (Fig. 2). A $\geqslant 3 \log$ decrease in bacterial count was reached with $<2 \mathrm{~h}$ of drug exposure. In contrast, piperacillin/tazobactam exhibited a bacteriostatic effect against $E$. coli in both mixed and pure culture, as defined by a $<2 \log$ decrease from the starting inoculum (Fig. 2). Although the MIC recorded for piperacillin/ tazobactam against the E. coli strain used in this study was within the sensitivity limits, the results obtained from the bacterial kill curve do not support this susceptibility pattern, as the antimicrobial concentrations used were at least 32-fold higher than the MIC. A similar discrepancy between in vitro antimicrobial activity obtained from the bacterial kill curve and susceptibility tests has been described and attributed to the inoculum effect (Klepser et al., 1996; Stearne et al., 2001; Hermsen et al., 2005).

Fig. 2. Time-kill curves of ertapenem $\left(\bullet, 8.0 \mu \mathrm{g} \mathrm{ml}^{-1}\right)$ and piperacillin/tazobactam $\left(\boldsymbol{\Lambda}, 64.0 / 8.0 \mu \mathrm{g} \mathrm{ml}^{-1}\right)$ against $E$. coli alone (a) and in mixed culture with $B$. fragilis (b). A growth control ( $\square$ ) and the LLA (dashed line) are shown.

König et al. (1998) demonstrated that the activity of piperacillin was diminished at least 128 -fold with high inocula of E. coli ATCC 25922, the same strain as used in this study. In addition, Thomson \& Moland (2001) demonstrated the in vitro sensitivity of piperacillin/ tazobactam to the inoculum effect of extended-spectrum $\beta$-lactamase-producing bacteria.

Table 2. Killing rates of E. coli ATCC 25922 and B. fragilis ATCC 25285 in pure or mixed culture during the first $2 \mathrm{~h}$ of drug exposure

\begin{tabular}{|lccc|}
\hline Antibiotic & \multicolumn{3}{c|}{ Killing rate $\left(\log _{\mathbf{1 0}}\right.$ c.f.u. $\left.\mathbf{h}^{-\mathbf{1}} \mathbf{~ m l}^{-\mathbf{1}}\right)$} \\
\cline { 2 - 4 } & E. coli & B. fragilis & $\begin{array}{c}\text { E. coli/B. } \\
\text { fragilis }\end{array}$ \\
\hline Ertapenem & -1.800 & -0.959 & $-2.045 /-1.751$ \\
Piperacillin/ & -0.386 & -1.048 & $-0.719 /-1.562$ \\
tazobactam & & & \\
\hline
\end{tabular}


In the present study, large numbers of actively growing cells $\left(\sim 10^{8}\right.$ c.f.u. $\left.\mathrm{ml}^{-1}\right)$ were used to simulate in vivo conditions, as abscesses contain high numbers of bacteria in a stationary phase of growth (Goldstein \& Snydman, 2004). These high inocula of E. coli might be responsible for the reduced activity of piperacillin/tazobactam, although this antimicrobial agent had been effective against the same high inocula of $B$. fragilis. Soriano et al. (1996) demonstrated therapeutic relevance of the inoculum effect using an experimental model of intraperitoneal infection by E. coli in non-neutropenic and neutropenic mice. Their results showed a better correlation between in vitro activity and efficacy when the MICs considered were those obtained with a large inoculum $\left(\sim 10^{8}\right.$ c.f.u. $\left.\mathrm{ml}^{-1}\right)$ instead of the standard inoculum $\left(\sim 10^{5}\right.$ c.f.u. $\left.\mathrm{ml}^{-1}\right)$. However, other studies suggest that inoculum effect is an artefact of in vitro susceptibility testing and is consequently of little clinical relevance (Craig et al., 2004). Thus the available literature is still controversial and inconclusive about the inoculum effect on the activity of antimicrobial agents. A caveat of the present study is that only a single strain of $B$. fragilis and E. coli was examined and it remains to be determined whether the findings of this investigation extend to other strains.

In summary, the results of the present study showed similar activities of ertapenem and piperacillin/tazobactam against $B$. fragilis ATCC 25285, alone or in mixed culture with E. coli. However, ertapenem was markedly more efficient than piperacillin/tazobactam against E. coli ATCC 25922, in both pure and mixed culture.

\section{ACKNOWLEDGEMENTS}

The study was supported by grants from Conselho Nacional de Desenvolvimento Científico e Tecnológico (CNPq), Fundação de Amparo à Pesquisa do Estado de Minas Gerais (FAPEMIG) and Merck Sharp \& Dohme. The authors are grateful to Luzia Rosa Resende and José Sérgio Barros de Souza for technical support. This work was partially presented at the 106th General Meeting of the American Society for Microbiology, Orlando, FL, USA, 21--25 May 2006.

\section{REFERENCES}

Betriu, C., Salso, S., Sanchez, A., Culebras, E., Gómez, M., Rodriguez-Avial, I. \& Picazo, J. J. (2006). Comparative in vitro activity and the inoculum effect of ertapenem against Enterobacteriaceae resistant to extended-spectrum cephalosporins. Int $J$ Antimicrob Agents 28, 1-5.

Craig, W. A., Bhavnani, S. M. \& Ambrose, P. G. (2004). The inoculum effect: fact or artifact? Diagn Microbiol Infect Dis 50, 229-230.

Dela Pena, A. S., Asperger, W., Kockerling, F., Raz, R., Kafka, R., Warren, B., Shivaprakash, M., Vrijens, F., Giezek, H. \& other authors (2006). Efficacy and safety of ertapenem versus piperacillin-tazobactam for the treatment of intra-abdominal infections requiring surgical intervention. Optimizing Intra-Abdominal Surgery with Invanz (OASIS)-I Study Group. J Gastrointest Surg 10, 567-574.

Goldstein, E. J. C. \& Snydman, D. R. (2004). Intra-abdominal infections: review of the bacteriology, antimicrobial susceptibility and the role of ertapenem in their therapy. J Antimicrob Chemother 53 (Suppl. 2), ii29-ii36.

Goldstein, E. J. C., Citron, D. M., Merriam, C. V., Warren, Y. \& Tyrrell, K. L. (2000). Comparative in vitro activities of ertapenem (MK-0826) against 1001 anaerobes isolated from human intra-abdominal infections. Antimicrob Agents Chemother 44, 2389-2394.

Heilmann, F. (1993). Ampicillin and ampicillin-sulbactam dilution tests with mixed cultures of Bacteroides fragilis, Escherichia coli and Enterococcus. Infection 21, 187-190.

Hermsen, E. D., Hovde, I. B., Sprandel, K. A., Rodvold, K. A. \& Rotschafer, J. C. (2005). Levofloxacin plus metronidazole administered once daily versus moxifloxacin monotherapy against a mixed infection of Escherichia coli and Bacteroides fragilis in an in vitro pharmacodynamic model. Antimicrob Agents Chemother 49, 685-689.

Jerman, B., Butala, M. \& Žgur-Bertok, D. (2005). Sublethal concentrations of ciprofloxacin induce bacteriocin synthesis in Escherichia coli. Antimicrob Agents Chemother 49, 3087-3090.

Klepser, M. E., Banevicius, M. A., Quintiliani, R. \& Nightingale, C. H. (1996). Characterization of bactericidal activity of clindamycin against Bacteroides fragilis via kill curve methods. Antimicrob Agents Chemother 40, 1941-1944.

König, C., Simmen, H. P. \& Blaser, J. (1998). Bacterial concentrations in pus and infected peritoneal fluid - implications for bactericidal activity of antibiotics. J Antimicrob Chemother 42, 227-232.

Muñoz Bellido, J. L., Muñoz Criado, S., García García, I., Alonso Manzanares, M. A., Gutiérrez Zufiaurre, M. N. \& García-Rodrigues, J. A. (1997). In vitro activities of $\beta$-lactam- $\beta$-lactamase inhibitor combinations against Stenotrophomonas maltophilia: correlation between methods for testing inhibitory activity, time-kill curves, and bactericidal activity. Antimicrob Agents Chemother 41, 2612-2615.

NCCLS (2003). Methods for Dilution Antimicrobial Susceptibility Tests for Bacteria that Grow Aerobically, 6th edn. Approved Standard. NCCLS document M7-A6. Wayne, PA: National Committee for Clinical Laboratory Standards.

NCCLS (2004). Methods for Antimicrobial Susceptibility Testing for Anaerobic Bacteria, 6th edn. Approved Standard. NCCLS publication M11-A6. Wayne, PA: National Committee for Clinical Laboratory Standards.

Pendland, S. L., Jung, R., Messick, C. R., Schriever, C. A. \& Patka, J. (2002). In vitro bactericidal activity of piperacillin, gentamicin, and metronidazole in a mixed model containing Escherichia coli, Enterococcus faecalis, and Bacteroides fragilis. Diagn Microbiol Infect Dis 43, 149-156.

Perry, C. M. \& Markham, A. (1999). Piperacillin/tazobactam: an updated review of its use in the treatment of bacterial infections. Drugs 57, 805-843.

Sader, H. S., Ferreira, A. T., Tosin, I. I., Gales, A. C., Keim, L. S., Carbadillo, J. M., Mello, S. J., Jr \& Tavares, W. (1998). Piperacillin/ tazobactam: evaluation of its in vitro activity against bacteria isolated in two Brazilian hospitals and an overview of its antibacterial activity, pharmacokinetic properties and therapeutic potential. Braz J Infect Dis 2, 241-255.

Schaumann, R., Goldstein, E. J., Forberg, J. \& Rodloff, A. C. (2005). Activity of moxifloxacin against Bacteroides fragilis and Escherichia coli in an in vitro pharmacokinetic/pharmacodynamic model employing pure and mixed cultures. J Med Microbiol 54, 749-753.

Solomkin, J. S. \& Konstantin, U. (2003). Intraabdominal sepsis: newer interventional and antimicrobial therapies for infected necrotizing pancreatitis. Curr Opin Crit Care 9, 424-427.

Solomkin, J. S., Yellin, A. E., Rotstein, O. D., Christou, N. V., Dellinger, E. P., Tellado, J. M., Malafaia, O., Fernandez, A., Choe, K. A. $\&$ other authors (2003). Ertapenem versus piperacillin/tazobactam in 
the treatment of complicated intraabdominal infections: results of a double-blind, randomized comparative phase III trial. Protocol 017 Study Group. Ann Surg 237, 235-245.

Soriano, F., Garcia-Corbeira, P., Ponte, C., Fernandez-Roblas, R. \& Gadea, I. (1996). Correlation of pharmacodynamic parameters of five $\beta$-lactam antibiotics with therapeutic efficacies in an animal model. Antimicrob Agents Chemother 40, 2686-2690.

Spangler, S. K., Jacobs, M. R. \& Appelbaum, P. C. (1997). Bactericidal activity of DU-6859a compared to activities of three quinolones, three $\beta$-lactams, clindamycin, and metronidazole against anaerobes as determined by time-kill methodology. Antimicrob Agents Chemother 41, 847-849.
Stearne, L. E., Kooi, C., Goessens, W. H., Bakker-Woudenberg, I. A \& Gyssens, I. C. (2001). In vitro activity of trovafloxacin against Bacteroides fragilis in mixed culture with either Escherichia coli or a vancomycin-resistant strain of Enterococcus faecium determined by an anaerobic time-kill technique. Antimicrob Agents Chemother 45, 243-251.

Thomson, K. S. \& Moland, E. S. (2001). Cefepime, piperacillintazobactam, and the inoculum effect in tests with extended-spectrum $\beta$-lactamase-producing Enterobacteriaceae. Antimicrob Agents Chemother 45, 3548-3554.

Wexler, H. M. (2004). In vitro activity of ertapenem: review of recent studies. J Antimicrob Chemother 53, ii11-ii21. 